\title{
Determinantes del gasto de bolsillo en salud de la población pobre atendida en servicios de salud públicos en Perú, 2010-2014
}

\author{
Margarita Petrera Pavone ${ }^{1}$ y Eduardo Jiménez Sánchez ${ }^{2}$
}

Forma de citar

Petrera Pavone M, Jiménez Sánchez E. Determinantes del gasto de bolsillo en salud de la población pobre atendida en servicios de salud púbicos en Perú, 2010-2014. Rev Panam Salud Publica. 2018;42:e20. https://doi.org/10.26633/RPSP.2018.20

RESUMEN Objetivo. El objetivo de este estudio fue averiguar qué factores afectan el gasto de bolsillo en servicios de salud prestados por el MINSA y los gobiernos regionales a la población en condiciones de pobreza monetaria relativa entre 2010 y 2014.

Materiales y métodos. Estudio transversal descriptivo econométrico con cortes anuales (2010-2014) a nivel nacional con información de encuestas de hogares. La variable dependiente fue el gasto de bolsillo en salud de las personas en situación de pobreza monetaria relativa para costear su atención médica en servicios de salud públicos. Las variables independientes fueron la afiliación al Seguro Integral de Salud (SIS), el nivel de gasto familiar per capita, el grupo de edad (0-5 años), ser mujer en edad fértil (15-49 años), ser persona adulta mayor (mayor de 60 años)), la carga familiar (cuyo indicador proxy fue el número de miembros en el hogar), y el nivel de complejidad del prestador de servicios.

Resultados. Sólo $5 \%$ de la población rural objeto de estudio fue atendida en centros de mayor nivel de complejidad (hospital) frente a 16\% de la del medio urbano. Los medicamentos concentraron el mayor gasto de hogares: $44 \%$ en los asegurados en el SIS y 62\% en los no asegurados (2014). El gasto de bolsillo en salud se asoció positivamente con no estar afiliado en el SIS, el mayor nivel de complejidad del prestador, el nivel del gasto familiar per capita y ser adulto mayor.

Conclusiones. La afiliación al SIS es una variable de política relevante para disminuir el gasto de bolsillo en hogares pobres. El menor gasto de bolsillo de la población rural pobre, especialmente vulnerable, está fuertemente asociado con el uso casi exclusivo de servicios provistos por prestadores del primer nivel de atención. Se necesitan políticas específicas de protección de la población adulta mayor en condiciones de pobreza. Para lograr la cobertura universal en salud, Perú necesita políticas más intensivas de protección financiera y de reestructuración de su oferta pública.

Palabras clave Seguro de salud; pobreza; gasto de bolsillo; cobertura universal; Perú.

En octubre de 2014, los países de la Región de las Américas acordaron firmar una resolución destinada a avanzar hacia el acceso y la cobertura universales de
Margarita Petrera. Correo electrónico: mpetrerap@gmail.com

Universidad Nacional Mayor de San Marcos, Lima Perú. salud en las próximas décadas. Para ello, establecieron como una de las cuatro grandes líneas estratégicas incrementar el financiamiento público y disminuir el gasto de bolsillo de los hogares (1). En Perú, el Acuerdo Nacional es un espacio 
de concertación para definir políticas de Estado aprobadas por consenso entre entidades del Gobierno, partidos políticos y organizaciones de la sociedad civil, a fin de establecer una ruta de crecimiento sostenible para el país en un marco de equidad social y de fortalecimiento de la democracia (2). En junio de 2015, uno de los los objetivos marcados de la Reforma de Salud para 2021 fue el fortalecimiento del Seguro Integral de Salud (SIS) como "El gran instrumento de universalización del acceso al cuidado y atención integral de la salud" (3).

El aseguramiento público en salud se inició en Perú en 1998 con el Seguro Escolar Gratuito y, desde 1999, benefició a escolares de colegios nacionales a través del Seguro Materno-Infantil en algunos ámbitos geográficos. Finalmente, ambos seguros se fusionaron dando lugar al Seguro Público de Salud (2001), que en 2002 se renombró como SIS. Su objetivo principal es "construir un sistema de aseguramiento público que financie y promueva el acceso con equidad de la población no asegurada a prestaciones de salud, dándole prioridad a los grupos vulnerables en situación de pobreza y pobreza extrema" (4).

Con la estrategia del Aseguramiento Universal en Salud, explicitada en la Ley del mismo nombre en el 2009 (5) y su reglamento en 2010 (6), el SIS pasó a ser el asegurador de régimen subsidiado enfocado a la población en condiciones de pobreza, con independencia del grupo de edad y financiado totalmente con recursos públicos. Los establecimientos públicos de salud del Ministerio de Salud (MINSA) y de los gobiernos regionales son los principales prestadores de los servicios de salud solicitados por los asegurados en el SIS, con lo que cabría esperar un importante efecto de dicha afiliación en la disminución de su gasto de bolsillo o gasto directo en salud. La Organización Mundial de la Salud (OMS) define este gasto como los honorarios, tarifas o cualquier tipo de pago efectuado por consulta médica, procedimientos de diagnóstico, tratamiento, con o sin receta médica, formal, informal o tradicional, ademas de los copagos y deducibles efectuados si es que se dispone de algún tipo de seguro médico (7).

Hay pruebas empíricas de que el SIS tuvo un efecto protector del acceso a servicios de salud para el año 2010 al demostrarse que la probabilidad de un afiliado de acceder a servicios de salud era tres veces mayor que la de un no afiliado $\mathrm{y}$, si bien no todos los afiliados al SIS que concurrían a la red nacional del MINSA obtenían en gratuidad total la atención, se observaba dentro de la población en condiciones de pobreza relativa un menor gasto de bolsillo en salud entre los afiliados al SIS respecto a los no afiliados entre 2004 y 2010 (8). Un efecto similar se notificó en las Cuentas Nacionales en Salud entre 2004 y $2012(9,10)$, aunque amenaza reducirse (9).

El $61 \%$ del gasto de bolsillo en Perú para costear una atención médica recibida de un prestador público de servicios de salud (el MINSA y los gobiernos regionales) se realiza en establecimientos privados cercanos (farmacias, centros de diagnóstico y laboratorios) (9).

Según la OMS, el gasto de bolsillo en salud es regresivo, excluyente, no solidario, y no permite anticipar la aparición de enfermedades o accidentes y afecta, principalmente, la salud de los pobres que no pueden pagar los servicios que necesitan (7). En promedio, en América Latina el gasto de los hogares asciende a $45 \%$ del gasto total en salud, un porcentaje mayor que el de los países de Europa y de la Organización para la Cooperación y el Desarrollo Económico (OCDE), lo que además de ser una de las principales fuentes de inequidad, limita la capacidad de los países para avanzar hacia la cobertura universal en salud (11). Aunque en Perú el gasto de bolsillo era menor (37\%), continuaba siendo la principal fuente de financiamiento de los servicios de salud en 2012 (9). Estudios a nivel mundial señalan que el gasto de bolsillo depende de la capacidad de pago, de aspectos demográficos, del desarrollo de enfermedades, de los tipos de servicios de salud que se prestan y de los cambios tecnológicos $(12,13)$. En siete países (Argentina, Brasil, Chile, Colombia, Ecuador, México y Uruguay) se encuentran tres características que elevan la magnitud de los gastos de bolsillo: ser jefe del hogar mayor de 65 años, la presencia de adultos mayores en el hogar y la hospitalización (14). En estudios realizados en México, se seleccionaron como variables explicativas del gasto de bolsillo la tenencia del Seguro Popular de Salud, el nivel de gasto per capita y la pertenencia a grupos específicos de edad (0-5 años, mujer en edad fértil, adulto mayor) (15, 16). Similares hallazgos se notificaron en Chile, aunque con un impacto decreciente en dichos grupos de edad $(17,18)$.
El objetivo de este estudio fue averiguar qué factores afectan el gasto de bolsillo en servicios de salud prestados por el MINSA y los gobiernos regionales de la población en condiciones de pobreza monetaria relativa entre 2010 y 2014.

\section{MATERIALES Y MÉTODOS}

Se realizó un estudio transversal descriptivo econométrico en todo el país con cortes anuales entre 2010 y 2014. En él se utilizó información secundaria obtenida de las bases de datos de dicho periodo de la Encuesta Nacional de Hogares (ENAHO). Esta encuesta, realizada por el Instituto Nacional de Estadística e Informática (INEI) desde 1995, es de dominio público y está dirigida a los hogares con objeto de explorar el nivel de vida de la población (19). La encuesta se hizo a las personas que vivían en viviendas particulares de las áreas urbana y rural del país. Los informantes fueron el jefe del hogar, el cónyuge, los perceptores de ingresos y las personas de 12 o más años de edad. El método empleado fue el de entrevista directa utilizando equipos móviles para captura de datos (PDA). El tipo de encuesta fue de derecho y el personal de campo estuvo compuesto por coordinadores departamentales, supervisores y encuestadores. La muestra fue del tipo probabilístico, de áreas, estratificada, multietápica e independiente en ámbito territorial de estudio. El nivel de confianza de las estimaciones muestrales fue de $95 \%$.

La población objeto del presente estudio fue una muestra compuesta por aquellas personas que vivían en condiciones de pobreza monetaria relativa (quintiles 1 y 2 del gasto familiar per cápita) y que, habiendo declarado que tenían la percepción de padecer una enfermedad o un accidente en salud, utilizaron los servicios de salud de prestadores públicos de salud del MINSA o de los gobiernos regionales y efectuaron algún gasto de bolsillo para pagar dicha atención. El tamaño de la submuestra seleccionada fue de 2149 (2010) y de 2807 personas (2014).

El gasto per capita se calculó a partir del gasto total de los hogares (alimentos, cuidado personas, educación salud, vivienda, etc.) estimado por el INEI y dividido por el número de miembros del hogar. El gasto per capita familiar se utilizó para dividir a la población según los quintiles de la distribución del gasto 
familiar per cápita, lo que, según la bibliografía (17), es una buena medida del bienestar económico. Se considera población en pobreza monetaria relativa a la situada en los dos primeros quintiles de dicha distribución.

Los criterios de inclusión fueron: a) personas que pertenecían a los quintiles 1 y 2 del gasto familiar per cápita, b) las que declararon tener la percepción de padecer una enfermedad o un accidente y que utilizaron los servicios de salud de los prestadores públicos del MINSA o de los gobiernos regionales en las últimas cuatro semanas o que fueron hospitalizadas en los últimos doce meses, y (c) las que declararon haber realizado algún gasto monetario de bolsillo para costear dicha atención, excluyendo a quienes obtuvieron gratuidad total (pago $=0$ ). $\mathrm{La}$ condición de afiliado al SIS se obtuvo de la misma ENAHO.

Los criterios de exclusión fueron: a) personas que pertenecían a los quintiles 3,4 y 5 del gasto familiar per cápita, b) las que no declararon tener la percepción de padecer una enfermedad o accidente, c) las que tenían un seguro de salud diferente del SIS, d) las que, habiendo declarado la percepción de enfermedad o accidente, no utilizaron los servicios de salud de prestadores públicos de salud del MINSA o de los gobiernos regionales en las últimas 4 semanas o no fueron hospitalizadas en los últimos 12 meses, y e) las que, habiendo utilizado los servicios de salud de los establecimientos públicos del MINSA o de los gobiernos regionales en las últimas 4 semanas o habiendo sido hospitalizadas en los últimos 12 meses, no efectuaron un gasto de bolsillo en servicios de salud.

La variable dependiente del estudio fue el gasto de bolsillo o pago directo realizado por las personas que viven en condiciones de pobreza relativa para pagar la atención recibida de los prestadores públicos del MINSA y de los gobiernos regionales para hacer frente a un problema de salud. Operativamente, se identificó el tipo de gasto (mayor a cero) pagado por algún miembro del hogar en medicamentos, insumos médicos, lentes ópticos, aparatos ortopédicos y terapéuticos, consulta médica, intervención médica en operaciones, honorarios de otros profesionales, servicio dental, análisis y servicios de laboratorio, rayos e imágenes, servicio de hospitalización, servicio de emergencia móvil, y copago por el uso del seguro de salud.
Esta variable no sigue una distribución normal ni en valor absoluto ni en logarítmico. Sin embargo, no es necesario relajar el supuesto de normalidad si el objetivo sólo es realizar la estimación, porque se tiene en cuenta la extensión del teorema del límite central y, por ello, las pruebas t de Student y F de Snedecor aún son válidas asintóticamente, es decir, en muestras grandes como la de este estudio $(21,22)$.

Las variables independientes fueron: la afiliación al SIS, el nivel de gasto familiar per capita, el grupo de edad (0-5 años, mujer en edad fértil (15-49 años), ser persona adulta mayor (mayor de 60 años)), la carga familiar (cuyo indicador proxy fue el número de miembros en el hogar), y el nivel de complejidad del prestador de servicios donde se efectuó la atención (su indicador proxy fue una variable dicotómica: hospital = mayor complejidad y centro/puesto de salud = menor complejidad).

Como $95 \%$ de la población rural fue atendida en establecimientos de menor complejidad, en la regresión no se pudo utilizar la variable ámbito urbano/rural donde habita la persona, debido a un problema potencial de colinearidad entre el nivel de complejidad y el ámbito. Siendo la población rural $61,5 \%$ del total de población de la muestra, se optó por privilegiar el nivel de complejidad al cual se accede (cuadro 1). Se hizo una estimación robusta, es decir, se calculó la verdadera matriz de varianzas y covarianzas para eliminar la posibilidad de tener perturbaciones no esféricas $(21,22)$.

Para estimar las asociaciones entre el gasto de bolsillo y las variables independientes escogidas, se construyó un modelo de regresión lineal multivariante. Las regresiones se realizaron individualmente para cada uno de los años del periodo comprendido entre 2010 y 2014. En los modelos se introdujeron los términos de interacción entre la afiliación al SIS y las otras variables independientes y posteriormente se excluyeron porque no fueron estadísticamente significativos.

\section{CUADRO 1. Distribución de la población de estudio por ámbito, Perú, 2014}

\begin{tabular}{lccc}
\hline & $\begin{array}{c}\text { Gasto familiar per capita anual } \\
\text { en salud (soles) }\end{array}$ & $\begin{array}{c}\text { Personas atendidas en } \\
\text { atención primaria (\%) }\end{array}$ & Población estudiada (\%) \\
\hline Urbana & 332 & 84,4 & 38,5 \\
Rural & 247 & 95,3 & 61,5 \\
Total nacional & 287 & 91,1 & 100,0 \\
\hline
\end{tabular}

Fuente: Instituto Nacional de Estadística e Informática, base de datos, Módulos Sumaria y Salud, ENAHO 2010-2014.
La especificación de la regresión es logarítmica para las variables continuas, lo que permite que sus parámetros se interpreten como una elasticidad, es decir, en qué medida una variación porcentual de la variable independiente se asocia con una variación porcentual de la variable dependiente. En el caso de las variables dicotómicas, los parámetros deben interpretarse como una semielasticidad, es decir, la presencia de las características (ser afiliado SIS, por ejemplo) se asocia con el cambio porcentual en el gasto en salud y es igual al parámetro estimado multiplicado por 100. Los análisis se realizaron con el paquete estadístico STATA versión 13

Al haber utilizado información secundaria de las bases de datos de la ENAHO, que son públicas y anónimas, no es posible revelar la identidad de las personas.

\section{RESULTADOS}

Aunque en general la población en condiciones de pobreza monetaria accedió en el periodo estudiado básicamente al primer nivel de atención (91,1\%), la rural accedió más (62 frente a 38,5\% la urbana\%) en 2014 y realizó menos consultas en establecimientos de mayor complejidad: sólo 5\% en 2014 mientras que en la población urbana este porcentaje ascendió a $16 \%$ (cuadro 1 ).

Las medias de las variables independientes de la muestra durante el periodo 2010-2014 revelan una disminución de la población menor de 5 años de 18 a 12\%, un aumento de los adultos mayores de 12 a $21 \%$ y una reducción del número de miembros del hogar de 5,5 a 5,0, una evolución que es compatible con la transición demográfica. Un elevado porcentaje de la población de la muestra estaba afiliada al SIS, lo que podría sugerir que la población en condiciones de pobreza monetaria relativa consulta preferentemente a los establecimientos públicos, aun cuando deba efectuar algún pago (cuadro 2).

En general, en el periodo 2010-2014, el gasto de bolsillo en soles constantes de 
las personas en condiciones de pobreza monetaria relativa muestra una tendencia hacia un ligero incremento, menor en los afiliados al SIS que en los no afiliados (cuadro 3). Expresado en dólares de paridad de poder adquisitivo (PPA) (23), y usando el tipo de cambio de 2014, en 2010 dicho gasto fue 161 \$US PPA para los afiliados y 180 \$US PPA para los no afiliados. Para 2014 estas cantidades ascendieron a 182 \$US PPA y 209 \$US PPA, respectivamente.

El medicamento fue el principal factor que explica el gasto de bolsillo en salud de la población en pobreza monetaria relativa. En 2014, del 100\% de gasto de bolsillo en atención de salud ofrecida por los prestadores del MINSA y de los gobiernos de las regiones, los afiliados al SIS gastaron $44 \%$ en medicamentos y los no afiliados, $62 \%$. No obstante, este tipo de gasto tiende a disminuir entre los afiliados SIS de 56,5 (2010) a 44,0\% (2014) (cuadro 4).

Los resultados de la regresión lineal multivariante logarítmica del gasto de bolsillo en salud de la población en condiciones de pobreza relativa en la atención prestada por el MINSA y los gobiernos regionales para cada uno de los años del periodo 2010-2014 muestran que la mayoría de las asociaciones entre las variables explicativas del modelo y el gasto de bolsillo son estadísticamente

CUADRO 2. Medias de las variables independientes de la muestra del estudio por año, Perú, 2010-2014

\begin{tabular}{cccccccc}
\hline Año & $\begin{array}{c}\text { Gasto familiar } \\
\text { per capita anual }\end{array}$ & $\begin{array}{c}\text { Niños } \\
\text { (0-5 años) }\end{array}$ & $\begin{array}{c}\text { Mujeres en } \\
\text { edad fértil }\end{array}$ & $\begin{array}{c}\text { Adultos } \\
\text { mayores }\end{array}$ & $\begin{array}{c}\text { Contar con } \\
\text { seguro del SIS }\end{array}$ & $\begin{array}{c}\text { Miembros } \\
\text { del hogar }\end{array}$ & $\begin{array}{c}\text { Personas atendidas } \\
\text { en un hospital }\end{array}$ \\
\hline 2010 & 2331 & 0,18 & 0,27 & 0,12 & 0,96 & 5,51 & 0,11 \\
2011 & 2533 & 0,17 & 0,28 & 0,15 & 0,95 & 5,36 & 0,12 \\
2012 & 2717 & 0,12 & 0,28 & 0,17 & 0,94 & 5,23 & 0,14 \\
2013 & 2938 & 0,12 & 0,28 & 0,19 & 0,94 & 5,18 & 0,12 \\
2014 & 3096 & 0,12 & 0,27 & 0,21 & 0,94 & 4,98 & 0,12 \\
\hline
\end{tabular}

Fuente: Instituto Nacional de Estadística e Informática, base de datos, Módulos Sumaria y Salud, ENAHO $2010-2014$. SIS = Seguro Integral de Salud.

CUADRO 3. Gasto de bolsillo' en salud de la población en situación de pobreza monetaria relativa según su afiliación, Perú, 2010-2014

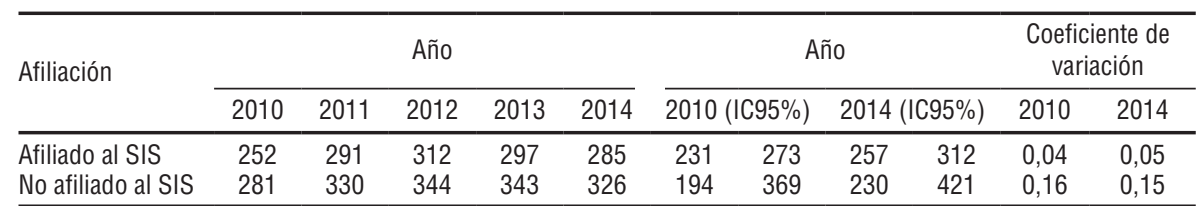

Fuente: Instituto Nacional de Estadística e Informática, base de datos, Módulos Sumaria y Salud, ENAHO 2010-2014.

${ }^{1}$ Deflactado por el Índice de Precios de Cuidado y Conservación de la Salud.

IC95\% = Intervalo de confianza de $95 \%$.

SIS = Seguro Integral de Salud. significativas (cuadro 5). Así, la afiliación al SIS se asocia con una reducción de dicho gasto entre 28,4 y $60,1 \%$. Ser atendido en un hospital antes que en una posta o centro de salud y disponer de un mayor ingreso en el hogar aumenta dicho gasto. Recibir atención en un hospital incrementa el gasto de bolsillo en salud entre 91,4 y $113,5 \%$, lo que se explica por el mayor nivel de complejidad de los servicios de salud prestados, que inducen un mayor gasto; esto ocurre más en las personas que viven en el medio urbano que en el rural. Cada aumento del gasto total del individuo (ser menos pobre) de $1 \%$ se asocia con un aumento del gasto de bolsillo en salud entre 0,6 y $1,1 \%$.

Ser menor de cinco años de edad aumenta el gasto de bolsillo, aunque esta asociación sólo fue estadísticamente significativa en 2010 (encontrarse en este grupo de edad está asociado con un aumento del gasto de bolsillo de 25,3\%). En las mujeres en edad fértil la asociación fue inversa en todos los años, aunque sólo fue estadísticamente significativa en 2012 y 2014, con reducciones del gasto de bolsillo de 13,4 y 20,7\% respectivamente. Ser adulto mayor se asoció con aumentos del gasto de bolsillo, si bien las asociaciones sólo fueron estadísticamente significativas en 2013 y 2014 (20,7 y 13,4\%, respectivamente). El aumento de un miembro en el hogar se asoció significativamente con ascensos del gasto de bolsillo en todos los años y dicho incremento varió entre 3,0\% (en 2010) y 6,6\% en 2014.

\section{DISCUSIÓN}

El hecho de que la población rural acceda menos que la urbana a servicios de

CUADRO 4. Composición porcentual del tipo de gasto de bolsillo personal en salud de la poblacion en situación de pobreza monetaria relativa por año y según su afiliación, Perú, 2010-2014

\begin{tabular}{|c|c|c|c|c|c|c|c|c|c|c|c|c|}
\hline \multirow{3}{*}{ Afiliación } & \multirow{3}{*}{$\begin{array}{l}\text { Tipo de gasto } \\
\text { Consulta }\end{array}$} & \multicolumn{5}{|c|}{ Año } & \multirow{2}{*}{\multicolumn{2}{|c|}{$\begin{array}{c}2010 \\
(I C 95 \%)\end{array}$}} & \multirow{2}{*}{\multicolumn{2}{|c|}{$\begin{array}{c}2014 \\
(I C 95 \%)\end{array}$}} & \multicolumn{2}{|c|}{ Coeficiente de variación } \\
\hline & & \multirow{2}{*}{$\begin{array}{r}2010 \\
6,3\end{array}$} & \multirow{2}{*}{$\begin{array}{r}2011 \\
6,7\end{array}$} & \multirow{2}{*}{$\begin{array}{r}2012 \\
6,9\end{array}$} & \multirow{2}{*}{$\begin{array}{r}2013 \\
5,8\end{array}$} & \multirow{2}{*}{$\frac{2014}{4,4}$} & & & & & 2010 & 2014 \\
\hline & & & & & & & 5,3 & 7,3 & 3,6 & 5,2 & 0,16 & 0,18 \\
\hline \multirow{4}{*}{ Afiliados SIS } & Medicamentos & 56,5 & 51,0 & 48,5 & 45,1 & 44,0 & 53,8 & 59,2 & 41,5 & 46,5 & 0,05 & 0,06 \\
\hline & Hospitalización/cirugía & 4,4 & 4,4 & 4,9 & 3,9 & 3,6 & 3,4 & 5,4 & 2,9 & 4,4 & 0,23 & 0,20 \\
\hline & Otros gastos ${ }^{1}$ & 32,8 & 37,8 & 39,7 & 45,2 & 48,0 & & & & & & \\
\hline & Consulta & 23,3 & 22,2 & 24,5 & 23,2 & 22,5 & 21,1 & 25,5 & 20,3 & 24,7 & 0,10 & 0,10 \\
\hline \multirow{3}{*}{ No afiliado SIS } & Medicamentos & 62,5 & 63,0 & 59,3 & 60,7 & 62,0 & 59,7 & 65,2 & 59,2 & 64,8 & 0,04 & 0,04 \\
\hline & Hospitalización/cirugía & 2,0 & 2,4 & 2,8 & 1,1 & 1,7 & 0,9 & 3,2 & 0,6 & 2,8 & 0,56 & 0,66 \\
\hline & Otros gastos ${ }^{1}$ & 12,2 & 12,5 & 13,4 & 15,1 & 13,8 & & & & & & \\
\hline
\end{tabular}

Fuente: Instituto Nacional de Estadística e Informática, base de datos, Módulos Sumaria y Salud, ENAHO 2010-2014.

IC = Intervalo de confianza.

SIS = Seguro Integral de Salud.

${ }^{1}$ Otros gastos comprenden análisis, diagnósticos y otras pruebas, , atención de parto, servicio dental y conexos, servicio oftalmológico, compra de lentes, control de embarazo, vacunas, control de niños sano, anticonceptivos, termómetro y otros insumos. 
CUADRO 5. Modelo de regresión del gasto de bolsillo personal en salud de la población en situación de pobreza monetaria relativa, Perú, 2010 - 2014

\begin{tabular}{|c|c|c|c|c|c|}
\hline Variables & 2010 & 2011 & 2012 & 2013 & 2014 \\
\hline $\begin{array}{l}\text { Gasto familiar per capita } \\
\text { en logaritmo }\end{array}$ & $\begin{array}{l}0,647^{\star \star \star} \\
(-0,0879)\end{array}$ & $\begin{array}{l}0,746^{\star \star *} \\
(-0,081)\end{array}$ & $\begin{array}{l}0,732^{\star * *} \\
(-0,0852)\end{array}$ & $\begin{array}{l}0,822^{\star * \star} \\
(-0,0789)\end{array}$ & $\begin{array}{l}1,068^{\star * \star} \\
(-0,0824)\end{array}$ \\
\hline Ser niño (0-5 años) & $\begin{array}{l}0,253^{* * *} \\
(-0,0781)\end{array}$ & $\begin{array}{r}0,0679 \\
(-0,078)\end{array}$ & $\begin{array}{c}0,126 \\
(-0,0885)\end{array}$ & $\begin{array}{c}0,133 \\
(-0,0821)\end{array}$ & $\begin{array}{c}0,109 \\
(-0,0831)\end{array}$ \\
\hline Ser mujer en edad fértil & $\begin{array}{l}-0,0718 \\
(-0,0791)\end{array}$ & $\begin{array}{l}-0,00511 \\
(-0,0731)\end{array}$ & $\begin{array}{l}-0,134^{*} \\
(-0,0753)\end{array}$ & $\begin{array}{l}-0,0559 \\
(-0,068)\end{array}$ & $\begin{array}{l}-0,207^{* * *} \\
(-0,0691)\end{array}$ \\
\hline Ser adulto mayor & $\begin{array}{c}0,12 \\
(-0,101)\end{array}$ & $\begin{array}{c}0,11 \\
(-0,09)\end{array}$ & $\begin{array}{c}0,153 \\
(-0,0945)\end{array}$ & $\begin{array}{l}0,207^{* * *} \\
(-0,0795)\end{array}$ & $\begin{array}{c}0,134^{*} \\
(-0,0795)\end{array}$ \\
\hline Tener seguro del SIS & $\begin{array}{l}-0,284^{* *} \\
(-0,123)\end{array}$ & $\begin{array}{l}-0,372^{\star \star *} \\
(-0,111)\end{array}$ & $\begin{array}{l}-0,437^{\star * *} \\
(-0,103)\end{array}$ & $\begin{array}{l}-0,601^{* * *} \\
(-0,0823)\end{array}$ & $\begin{array}{l}-0,374^{* * *} \\
(-0,0937)\end{array}$ \\
\hline $\begin{array}{l}\text { Número de miembros } \\
\text { del hogar }\end{array}$ & $\begin{array}{c}0,0302^{\star *} \\
(-0,0141)\end{array}$ & $\begin{array}{l}0,0640^{\star * *} \\
(-0,0132)\end{array}$ & $\begin{array}{l}0,0361^{\star *} \\
(-0,0149)\end{array}$ & $\begin{array}{l}0,0409^{* * *} \\
(-0,013)\end{array}$ & $\begin{array}{l}0,0655^{\star * *} \\
(-0,014)\end{array}$ \\
\hline Ser atendido en hospital & $\begin{array}{l}1,075^{\star \star \star} \\
(-0,101)\end{array}$ & $\begin{array}{l}1,055^{\star \star \star} \\
(-0,0894\end{array}$ & $\begin{array}{l}1,135^{\star \star \star} \\
(-0,0907)\end{array}$ & $\begin{array}{l}0,914^{\star \star \star} \\
(-0,0895)\end{array}$ & $\begin{array}{l}1,012^{\star \star \star} \\
(-0,0846)\end{array}$ \\
\hline Constante & $\begin{array}{c}-0,501 \\
(-0,713)\end{array}$ & $\begin{array}{l}-1,358^{\star \star} \\
(-0,675)\end{array}$ & $\begin{array}{l}-1,099 \\
(-0,706)\end{array}$ & $\begin{array}{l}-1,775^{\star \star \star} \\
(-0,662)\end{array}$ & $\begin{array}{l}-4,043^{\star * *} \\
(-0,693)\end{array}$ \\
\hline $\begin{array}{l}\text { Tamaño de muestra } \\
\text { R-cuadrado }\end{array}$ & $\begin{array}{l}2,149 \\
0,099\end{array}$ & $\begin{array}{l}2,573 \\
0,108\end{array}$ & $\begin{array}{l}2,185 \\
0,12\end{array}$ & $\begin{array}{l}2,931 \\
0,099\end{array}$ & $\begin{array}{l}2,807 \\
0,134\end{array}$ \\
\hline
\end{tabular}

Fuente: Instituto Nacional de Estadística e Informática, base de datos, Módulos Sumaria y Salud, ENAHO 2010-2014. Entre paréntesis aparecen las estimaciones robustas de los errores estándar.

${ }^{\star * *} P<0,01,{ }^{\star *} P<0,05, P<0,1$.

salud de mayor complejidad indica que en el caso peruano la ruralidad es un determinante social de exclusión. Por otro lado, la observación que $61 \%$ del gasto de bolsillo de quien acude a un prestador público acaba completándose en la oferta privada está vinculado con la observación de la Defensoría del Pueblo que señala carencias en la disponibilidad de servicios, insumos y pruebas diagnósticas en los establecimientos públicos, incluso de aquellos que figuran en el Plan Esencial de Servicios de Salud (PEAS) y debieran ser de otorgamiento obligatorio (24). En el 2016, el financiamiento ejecutado del SIS era sólo 13\% del gasto del MINSA y los gobiernos regionales (25), un monto totalmente insuficiente para asegurar una cobertura efectiva de los servicios financiados por el SIS. Aunque al inicio de su funcionamiento en 2002, y gracias a la existencia de una capacidad ociosa, el SIS financiaba sólo el gasto corriente en medicamentos e insumos, con el agotamiento de dicha capacidad, el limitado financiamiento se utilizó también para cubrir recursos humanos y gastos en infraestructura, lo que agravó la situación financiera y convirtió en urgente la necesidad de debatir el papel financiador del SIS,

Aunque el beneficio de la afiliación al SIS redujo el peso del gasto en medicamentos dentro del gasto de bolsillo
$-44,0 \%$ en afiliados frente a $62,0 \%$ en no afiliados-, este beneficio resulta insuficiente. En otros países se han notificado porcentajes similares, como en México $(41,3 \%)$ con el Seguro Popular de Salud en $2008(15,16)$ y en 2007 en Chile: en el grupo más pobre (A) de los afiliados a FONASA fue $40 \%$ y en los no afiliados, $73,8 \%$ (18). En el caso chileno, el seguro podría estar cubriendo predominantemente medicamentos de bajo costo y, aunque se beneficie un alto porcentaje de afiliados, el efecto sobre la protección financiera es limitado (18).

Si bien la afiliación al SIS se asocia con un descenso del gasto de bolsillo, el modelo de regresión muestra que lo aumenta el nivel de complejidad del establecimiento donde se consulta, lo que a su vez es un obstáculo para el acceso de la población rural por su menor capacidad de gasto. En los adultos mayores, un grupo poblacional que puede estar desamparado y encontrarse en condiciones de pobreza, el riesgo de exclusión aumenta notablemente. Los resultados obtenidos son compatibles con los descritos a escala mundial para la población general $(12,13)$. También lo son con los de los estudios realizados para Argentina, Brasil, Chile, Colombia, Ecuador, México y Uruguay, en los cuales se observó que ser jefe del hogar mayor de 65 años y la presencia de adultos mayores elevan los gastos de bolsillo. Por otra parte, la presencia de menores de 5 años en el hogar aumenta el gasto de bolsillo en Chile y México y lo disminuye en Argentina, que tiene planes materno-infantiles preferenciales (14). Asimismo, en los estudios realizados en México las variables explicativas del gasto de bolsillo fueron estár afiliado al Seguro Popular de Salud, el nivel de gasto per capita y la pertenencia a grupos específicos de edad (0-5 años, mujer en edad fértil y adulto mayor) (16), a semejanza de los realizados en Chile, aunque en este país el impacto del gasto en dichos grupos de edad fue decreciente (17).

Es posible que el efecto positivo sobre el gasto de los menores de 5 años observado en 2010, que desapareció en los años siguientes, se deba a la mejora progresiva de los programas que se dirigen a este grupo de edad. El ligero efecto positivo del tamaño del hogar (carga familiar) en el gasto de bolsillo podría sugerir que, a medida que aumenta el número de miembros por hogar, también lo hace la probabilidad de enfermarse.

Las limitaciones de este estudio parten de las restricciones de la ENAHO: 1) las originadas por el tamaño de la muestra y por la no respuesta en el rubro de gasto en el establecimiento de salud; 2) como en toda encuesta de hogares, puede haberse introducido un sesgo de recuerdo y, para controlarlo, la encuesta acotó el tiempo a las últimas 3 semanas para la consulta y al último año para la hospitalización, 3) dado que la encuesta incluyó a hogares a través de la visita a viviendas particulares y sus ocupantes, excluyó a los miembros de las fuerzas armadas que residen en cuarteles, campamentos, barcos y en otros lugares. También se excluyó a los residentes en viviendas colectivas (hoteles, hospitales, asilos y claustros religiosos, cárceles, etc.). No se encuentran disponibles otros estudios referidos al gasto en salud efectuados a partir de encuestas realizadas en consulta en establecimientos públicos, lo que se convierte en la principal fortaleza de la presente investigación.

Se concluye que el insuficiente financiamiento del SIS es una de las causas del gasto de bolsillo de sus afiliados, lo que debería obligar a debatir al más alto nivel el papel específico del SIS como fondo financiador y a acometer una profunda reforma financiera, no sólo para aumentar el financiamiento, sino para mejorar su organización y la transparencia. Además, la oferta 
sanitaria pública es limitada y requiere una reconversión que supere los retos que supone aumentar los recursos humanos, dotar las tecnologías e infraestructuras necesarias $y$, sobre todo, gestionar todo ello para ofrecer a la población los servicios que necesita. Un grupo especialmente vulnerable es el de los adultos mayores, que aumentará Estrategia para el Acceso Universal a la Salud y la Cobertura Universal de Salud. Washington, DC: OPS; 2014. Disponible en http://www.paho.org/uhexchange/index. php/es/uhexchange-documents/informacion-tecnica/27-estrategia-para-el-acceso-universal-a-la-salud-y-la-cobertura-universal-de-salud/file Acceso el 30 de diciembre de 2017.

2. Secretaría Ejecutiva del Acuerdo Nacional. Acuerdo Nacional. Lima: Secretaría Ejecutiva del Acuerdo Nacional; 2015. Disponible en: http:/ / acuerdonacional.pe/politicas-de-estado-del-acuerdonacional/definicion/ Acceso el 15 de octubre de 2017. Acceso el 30 de diciembre de 2017.

3. Secretaría Ejecutiva del Acuerdo Nacional. Acuerdo Nacional. Los objetivos de la Reforma de Salud 2015. Lima: Secretaría Ejecutiva del Acuerdo Nacional; 2015. Disponible en: http:/ / acuerdonacionalperu.tripod.com Acceso el 30 de diciembre de 2017.

4. Ministerio de Salud. Ley $27657 / 2002$ de 28 de enero. Lima: Diario Oficial El Peruano; 2002;216517. Disponible en: http://www. minsa.gob.pe/dgsp/archivo/LeyMarco. pdf Acceso el 30 diciembre de 2017.

5. Ley 29344. Ley Marco de Aseguramiento Universal en Salud. Lima: Diario Oficial El Peruano; 2009:394077. Disponible en: http:/ / diariooficial.elperuano.pe/ Normas Acceso el 30 de diciembre de 2017.

6. Reglamento de la Ley Marco de Aseguramiento Universal en Salud. Decreto Supremo 0082010-SA/2010 de 2 de abril. Lima: Diario Oficial El Peruano; 2010:416633. Disponible en: http://www. minsa.gob.pe/dgsp/archivo/LeyMarco. pdf Acceso el 30 de diciembre de 2017.

7. Organización Mundial de la Salud. La financiación de los sistemas de salud. Ginebra: OMS; 2010. Disponible en: http:/ / apps.who.int/iris/bitstream/10665/ 44373/1/9789243564029_spa.pdf Acceso el 30 de diciembre de 2017.

8. Petrera M. Aseguramiento en salud en el Perú ¿Existe un efecto inclusivo del Seguro Público en Salud? Berlín: Editorial Académica Española; 2012.

9. Ministerio de Salud. Cuentas Nacionales en Salud. Perú: 1995-2012. Lima: MINSA; 2015. Disponible en: http://bvs.minsa. gob.pe:81/local/MINSA/3248.pdf Acceso el 30 de diciembre de 2017. a medida que se avance en la transición demográfica.

Agradecimiento. Los autores agradecen los comentarios recibidos de la economista Lorena Prieto Toledo.

Financiación. Este estudio no recibió financiación.

\section{REFERENCIAS}

10. Petrera M. Reflexiones para la política sectorial de salud en Perú a partir de las Cuentas Nacionales de Salud. Economía (PUCP). 2016;39(78):35-65. Disponible en: http:/ / revistas.pucp.edu.pe/index.php/ economia/article/view/16513 Acceso el 30 de diciembre de 2017.

11. Titelman D, Cetrángolo O, Acosta OL. La cobertura universal de salud en los países de América Latina: cómo mejorar los esquemas basados en la solidaridad. MEDICC Review. 2015;17(Supl):s68-s72.

12. Xe K, Saksena P, Holly A. The determinants of health expenditure. A CountryLevel Panel Data Analysis. Geneva: WHO; 2011. (Working Paper.) Disponible en: who.int/health_financing/documents / report_en_11_deter-he.pdf Acceso el 30 de diciembre de 2017.

13. Xu K. Distribución del gasto en salud y gastos catastróficos. Ginebra: Organización Mundial de la Salud; 2005. (Discussion paper No. 2.) Disponible en: http://apps. who.int/iris/bitstream/10665/85626/1/ EIP_HSF_DP.05.2_spa.pdf Acceso el $30 \mathrm{de}$ diciembre de 2017.

14. Perticara M. Incidencia de los gastos de bolsillo en salud en siete países latinoamericanos. Santiago de Chile: CEPAL-PNUD; 2008. (Serie Políticas Sociales 141.) Disponible en: http://www.cepal.org/ es/publicaciones/6146-incidencia-gastosbolsillo-salud-siete-paises-latinoamericanos Acceso el 30 de diciembre de 2017.

15. Gakidou E, Lozano R, González-Pier E, Abbot-Klanffter J, Barofky JT, BrysonKahn C, et al. Evaluación del impacto de la Reforma Mexicana de salud 2001-2006: Un informe inicial. Salud Publica Mex. 2007;49(Supl 1):S88-S109. Disponible en: http:/ / www.scielo.org.mx/scielo.php?script=sci_arttext\&pid=S0036-36342007000 700011 Acceso el 30 de diciembre de 2017.

16. Knaul FM, González-Pier E, Gómez-Dantés $\mathrm{O}$, García-Junco D, Arreola-Ornelas H, Barraza-Llórens M, et al. Hacia la cobertura universal en salud: protección social para todos en México. Salud Publica Mex. 2013. 55(2):207-35. Disponible en: http://www. scielosp.org/pdf/spm/v55n2/v55n2a13. pdf Acceso el 30 de diciembre de 2017.

17. Cid C, Prieto L. El gasto de bolsillo en salud: El caso de Chile 1997 y 2007. Rev Panam Salud Publica. 2012;31(4):310-16. Disponible en: http://www.scielosp.org/ pdf/rpsp/v31n4/a07v31n4.pdf Acceso el 30 de diciembre de 2017.
Conflictos de interés. Los autores declaran no tener conflictos de interés.

Declaración. Las opiniones expresadas en este manuscrito son responsabilidad del autor y no reflejan necesariamente los criterios ni la política de la RPSP/ PAJPH y/o de la OPS.

18. Castillo-Laborde C, Villalobos P Caracterización del gasto de bolsillo en salud en Chile: Una mirada a dos sistemas de protección. Rev Med Chile. 2013; 141(11):1456-63. Disponible en: http://dx. doi.org/10.4067/S0034-9887201300110 0013 Acceso el 30 de diciembre de 2017.

19. Instituto Nacional de Estadística e Informática. Sistema de documentación virtual de investigaciones estadísticas. Lima: INEI; 2017. Disponible en: http:// webinei.inei.gob.pe/anda_inei/index. $\mathrm{php} / \mathrm{catalog} / 195$ Acceso el 30 de diciembre de 2017

20. Instituto Nacional de Estadística e Informática. Metodología para la medición de la pobreza. Lima: INEI; 2000. (Colección Metodologías Estadísticas.) Disponible en: https:/ /www.inei.gob.pe/ media/MenuRecursivo/metodologias/ pobreza01.pdf Acceso el 30 de diciembre de 2017.

21. Gujarati D. Econometría, $5^{a}$ ed. Bogotá: Mc Graw Hill; 2010:318.

22. Cameron A, Trivedi P. Microeconometrics: Methods and Applications. Cambridge: Cambridge University Press; 2005:80-1.

23. Fondo Monetario Internacional. Base de datos del documento Perspectivas de la Economía Mundial. Washington, DC: FMI; 2016.

24. Defensoría del Pueblo. Camino al Aseguramiento Universal en Salud (AUS). Resultados de la supervisión nacional a hospitales. Informe Defensorial No. 161. Lima: Defensoría del Pueblo; 2013. Disponible en: http://www2.congreso. gob.pe/sicr/cendocbib/con4_uibd.nsf / 9D2A7AFD45EA318905257BCE0078F1E7/ \$FILE/2.informe-161.pdf Acceso el 30 de diciembre de 2017.

25. Ministerio de Economía y Finanzas. Portal de Transparencia Económica, Consulta Amigable. Lima: Ministerio de Economía y Finanzas. Disponible en: http://www. minsa.gob.pe/portal/Comisiones / Transferencia/plan.asp Acceso el 30 de diciembre de 2017.

Manuscrito recibido el 21 de septiembre de 2017. Aceptado para publicación, tras revisión, el 11 de diciembre de 2017. 
ABSTRACT

Determinants of out-ofpocket spending on health among the poor population served by public health services in Peru, 2010-2014

Keywords
Objective. To ascertain which factors affect out-of-pocket spending on health services provided by the Ministry of Health of Peru and regional governments to the population living in relative poverty between 2010 and 2014.

Materials and methods. Cross-sectional, descriptive, nationwide, econometric, yearon-year (2010-2014) study using information from household surveys. The dependent variable was out-of-pocket spending by people in relative poverty to pay for medical care at public health services. The independent variables were affiliation to the Comprehensive Health Insurance (SIS) scheme, level of family expenditure per capita, age group 0-5 years, being a woman of childbearing age (15-49 years), being an older adult (over age 60 years), family burden (using the number of members of the household as a proxy indicator), and the level of complexity of the service provider.

Results. Only 5\% of the rural population in the study were treated at higher-complexity facilities (hospitals), compared to $16 \%$ of the population in urban centers. Drugs accounted for the majority of household expenditures: $44 \%$ among those insured via SIS and 62\% among the uninsured (2014). Out-of-pocket spending on health was positively associated with not being insured via SIS, higher level of provider complexity, level of family spending per capita, and being an older adult.

Conclusions. Comprehensive Health Insurance coverage is a relevant policy variable to reduce out-of-pocket spending in poor households. The lower out-of-pocket spending among the rural poor-an especially vulnerable group-was strongly associated with near-exclusive use of services delivered by primary health care providers. Specific policies are needed to protect older adults living in poverty. To achieve universal health coverage, Peru needs to implement more intensive financial protection policies and restructure its public service offering.

Health insurance; poverty; out-of pocket expenditures; universal coverage; Peru.
RESUMO

\section{Determinantes da despesa por conta própria em saúde da população pobre atendida em serviços públicos de saúde, Peru, 2010-2014}

Palavras-chave
Objetivo. Examinar os fatores que influem na despesa por conta própria em serviços de saúde prestados pelos governos federal (Ministério da Saúde) e regionais à população em situação de relativa pobreza entre 2010 e 2014.

Materiais e métodos. Estudo transversal descritivo econométrico com recortes anuais (2010-2014) realizado em nível nacional com dados de pesquisas domiciliares. A variável dependente foi despesa por conta própria em saúde de pessoas em situação de relativa pobreza para custear o atendimento médico em serviços públicos de saúde. As variáveis independentes foram ser segurado do Seguro Integral de Saúde (SIS) da rede pública, despesa familiar per capita, faixa etária (0-5 anos), ser mulher em idade reprodutiva (15-49 anos), ser idoso (acima de 60 anos), carga familiar (cujo indicador substituto foi o número de pessoas por domicílio) e nível de complexidade do prestador de serviços.

Resultados. Apenas 5\% da população rural estudada foram atendidos em centros com nível de complexidade mais alto (hospitais) em comparação a $16 \%$ da população urbana. Os medicamentos representaram o maior gasto das famílias: $44 \%$ para os segurados do SIS e $62 \%$ para não segurados (2014). A despesa por conta própria em saúde foi positivamente associada a não ser segurado do SIS, nível de complexidade mais alto do prestador de serviços, nível de gasto familiar per capita e ser idoso.

Conclusões. Ser segurado do SIS é uma variável de política importante para reduzir a despesa por conta própria em famílias pobres. A menor despesa por conta própria na população rural pobre, particularmente vulnerável, está muito associada ao uso quase exclusivo de serviços de atenção primária. Fazem-se necessárias políticas específicas de proteção da população idosa vivendo em situação de pobreza. Para alcançar a cobertura universal de saúde, o Peru precisa de políticas mais firmes de proteção financeira e reestruturação da oferta pública.

Seguro Saúde; pobreza; despesas do próprio bolso; cobertura universal; Peru 